\title{
Helium observation in the Martian ionosphere by an X-ray ultraviolet scanner on Mars orbiter NOZOMI
}

\author{
M. Nakamura ${ }^{1}$, K. Yamashita ${ }^{2}$, I. Yoshikawa ${ }^{3}$, K. Shiomi ${ }^{1}$, A. Yamazaki ${ }^{1}$, S. Sasaki $^{4}$, Y. Takizawa ${ }^{1}$, \\ M. Hirahara ${ }^{5}$, W. Miyake ${ }^{6}$, Y. Saito ${ }^{3}$, and S. Chakrabarti ${ }^{7}$ \\ ${ }^{1}$ Earth and Planetary Science, University of Tokyo, Tokyo 113-0033, Japan \\ ${ }^{2}$ Graduate School of Science, Nagoya University, Aichi 464-8601, Japan \\ ${ }^{3}$ Institute of Space and Astronautical Science, Kanagawa 229-0022, Japan \\ ${ }^{4}$ Geological Institute, University of Tokyo, Tokyo 113-0033, Japan \\ ${ }^{5}$ College of Science, Rikkyo University, Tokyo 171-8501, Japan \\ ${ }^{6}$ Communications Research Laboratory, Tokyo 184-8795, Japan \\ ${ }^{7}$ Boston University, U.S.A.
}

(Received August 8, 1997; Revised July 27, 1998; Accepted September 21, 1998)

We have built an X-ray ultraviolet (XUV) scanner on board Mars orbiter NOZOMI (Planet-B). This scanner has the He I and II emissions from the Martian atmosphere and ionosphere as its main target. These EUV emissions provide important information for the study of both Martian geological history and the interaction between solar wind and the Martian ionosphere. The XUV scanner will be operated in the parking orbit around the earth and also in the transfer orbit to Mars, where the terrestrial plasmasphere and interplanetary emissions will be studied.

\section{Introduction}

Japan's first Mars orbiter NOZOMI (Planet-B) was built by the Institute of Space and Astronautical Science (ISAS) of Japan. Its main scientific objective is to study the plasma environment around Mars, especially the interaction between the solar wind and the non-magnetized plasma. NOZOMI was successfully launched on July 4, 1998 from Kagoshima Space Center in Kyushu, Japan, by ISAS's solid propellant rocket $\mathrm{M}-\mathrm{V}-3$. NOZOMI will stay in the parking orbit around the earth for 6 months, leaving the earth in December 1998. It will arrive in Mars in October, 1999, after a one-year cruise.

The X-ray ultraviolet (XUV) scanner on board NOZOMI will measure the abundance and distribution of both helium gas and ion around Mars. Helium gas resonantly scatters the $58.4 \mathrm{~nm}$ (He I emission), and helium ion scatters the $30.4 \mathrm{~nm}$ (He II emission) EUV emissions from the sun. The XUV scanner will detect these scattered EUV emissions. All these measurements, which will be carried out from the outside of the Martian atmosphere, will allow us to decide the total abundance and the height distribution of helium gas and ions.

No observations of helium gas have ever been made in previous Mars missions, and helium ion was measured only by Phobos 2 (Barabash and Norberg, 1994; Barabash et al., 1995). On NOZOMI, these components are measured in situ by a neutral gas mass spectrometer and plasma instruments (Norberg et al., 1998). The XUV scanner and these instruments will make complementary observations, as the former gives information about global gas and plasma distribution, while the latter ones give local information.

Copy right $(\mathcal{C}$ The Society of Geomagnetism and Earth, Planetary and Space Sciences (SGEPSS); The Seismological Society of Japan; The Volcanological Society of Japan; The Geodetic Society of Japan; The Japanese Society for Planetary Sciences.

\section{Scientific Objectives}

\subsection{Helium abundance on Mars}

The measurement of helium abundance is important in order to know the internal activity of Mars, which has been debated for many years. In 1993, the EUV telescope on the Extreme Ultraviolet Explorer satellite (EUVE) was pointed to Mars for 3 days, measuring a $58.4 \mathrm{~nm}$ scattered emission of Martian helium gas (Krasnopolsky et al., 1994). Even though the signal was faint, the result seems to support the existence of helium gas. Krasnopolsky et al. (1994) tried to relate observed 4-He abundance with degassing from Mars, assuming continuous steady-state degassing. On earth, continuous degassing at mid-oceanic ridges can supply helium (most of $3-\mathrm{He}$ and $20-30 \%$ of $4-\mathrm{He}$ of the earth's total supply) into the atmosphere. A large amount of radiogenic 4-He can also be degassed from the continental crust through magmatic and hydrothermal processes. On Mars, however, continuous degassing cannot be readily assumed. Geomorphological and meteoritical studies suggest that there was young Martian volcanic activity, but there is no evidence of a continuous degassing source such as the mid-oceanic ridges occurring on earth. Neither are there currently active plume-type volcanoes or fumaroles on Mars. To explain atmospheric helium by volcanism, the time scale of intermittency of volcanism should be shorter than the residence time of helium in the atmosphere. Impact cratering would disturb crustal materials and supply radiogenic helium into the atmosphere. Since the size distribution of impactors should extend to the small (i.e., meteorite) size, outgassing due to impacts may be continuous. Using the crater density model and assuming that helium could degas from a volume as large as ten times the volume of a crater, $10-30 \mathrm{~kg} / \mathrm{yr}$ is introduced as the degassing rate. As the time scale for supplying $10^{10} \mathrm{~kg}$ helium is $10^{9} \mathrm{yr}$ 
(Sasaki and Tajika, 1995), impact cratering cannot be a sufficient degassing source. If a significant amount of 4-He is detected in the Martian atmosphere by the XUV scanner, there should be ongoing underground magmatic activities or hydrothermal circulation, transporting radiogenic helium into the atmosphere.

\subsection{Helium ions around Mars}

The ionization process of helium gas and its escape from the ionosphere to interplanetary space are some of our interests. As described in the previous section, helium gas of planetary origin produced in the inner part of Mars may degas into the atmosphere by way of planetary inner activities and diffuse into the atmosphere. Some of this gas is ionized through various processes, such as photoionization and electron impact ionization, and forms a part of the Martian ionosphere. In the top region of the ionosphere, such ionized particles are swept away by the interplanetary magnetic field (IMF) through interaction between the solar wind and the Martian ionosphere. These pick-up ions gyrate around the IMF in a frame moving with the solar wind, and in the rest frame they have a velocity ranging from zero to twice the solar wind speed and spread toward the tail region. This configuration is illustrated briefly in Luhmann and Brace (1991) and Luhmann and Kozyra (1991). Such a measurement is important in the study of the interaction between the solar wind and the Martian ionosphere.

The in situ measurement of helium ion in the Martian ionosphere was conducted only by the Phobos 2 mission (Barabash and Norberg, 1994; Barabash et al., 1995). They observed helium ion particles at 9,600 km from the Martian center and measured two typical helium ions, i.e., $10-20 \mathrm{keV}$ high-energy ions, which are equivalent to twice the solar wind speed, and 300-800 eV low-energy ions. They concluded that the high-energy ions indicate ion escape from Mars through a pick-up process and that the low-energy ions may have escaped as a result of acceleration by the electric field.

With the XUV scanner, we will try to take an image of the Martian ionosphere. This will prove difficult because the expected He II emission is very faint due to the thin Martian atmosphere and the absence of a magnetic field keeping the helium ions in the plasmasphere around the earth. Though under such a restriction, we still have a good chance of detecting the He II emission around Mars when we have enough accumulation of time, which we will discuss in Section 4. We are aware of the poor temporal and spatial resolution of our observation. However, we consider this is a worthwhile endeavor, as it will be the first optical helium ion observation in Mars. By means of the comparison between the helium ion and the helium gas images, we will have information on the formation process of the Martian ionosphere, including the ionization rate and seasonal variation. In case the cold helium ions are richer than we expect, the observation will have better time and spatial resolution.

\subsection{Terrestrial plasmasphere}

NOZOMI has been put into a parking orbit around the earth for 6 months before its insertion into the transfer orbit to Mars. The XUV will be turned on there to measure the helium ion distribution of the terrestrial plasmasphere. Such an observation from the distance of several earth radii has not been done so far. The global image of the plasmasphere will give information on its formation process.

In our understanding of this formation process, some problems still remain unsolved. During the refilling phase of the plasmasphere formation, plasma should be supplied from the conjugate ionospheres through field-aligned supersonic flows. The question is how the diffusive equilibrium state is realized under such a supersonic flow. Banks et al. (1971) proposed that fast ion streams are thermalized by shocks during the early stage of the refilling process, where the shock formation occurs as a result of the electrostatic coupling between counter-streaming ion beams interpenetrating to the equator. However, this coupling occurs under a very restrictive plasma condition, and the verifications of such conditions have not been done yet. This is one of the targets which the visualization of the plasmasphere by the XUV scanner can approach.

\subsection{Interplanetary He I and II emissions}

The interstellar helium gas penetrating into the solar system can be another target. The He I emission should be faint because helium can have neutral particles only in the region far away from the sun; otherwise, those particles are photo-ionized. There is sufficient time-approximately a year-for data accumulation in the cruise phase to Mars, and a statistical study will reveal the abundance of such penetrating interstellar helium gas.

As soon as they are photo-ionized, becoming helium ions, these ions are picked up by the IMF moving with the solar wind. In the frame moving with the solar wind, the pick-up ions make a gyration motion. When this motion is observed in a rest frame, the pick-up ions make a motion as shown in Fig. 1 when the IMF is perpendicular to the solar wind velocity. Most of the particles move away from the sun very fast and do not scatter solar EUV emissions due to the Doppler shift. However, in the circles shown in the figure, particle velocity is nearly zero, and the pick-up ions can resonantly scatter the EUV emission. Thus, these interplanetary pick-up ions can also be our target

The $\alpha$ particles $\left(\mathrm{He}^{++}\right)$in the solar wind have a charge exchange with interstellar hydrogen atoms, becoming helium ions $\left(\mathrm{He}^{+}\right)$. However, these helium ions are flowing with the solar wind speed, so we do not expect them to scatter the solar EUV emission effectively due to the Doppler shift.

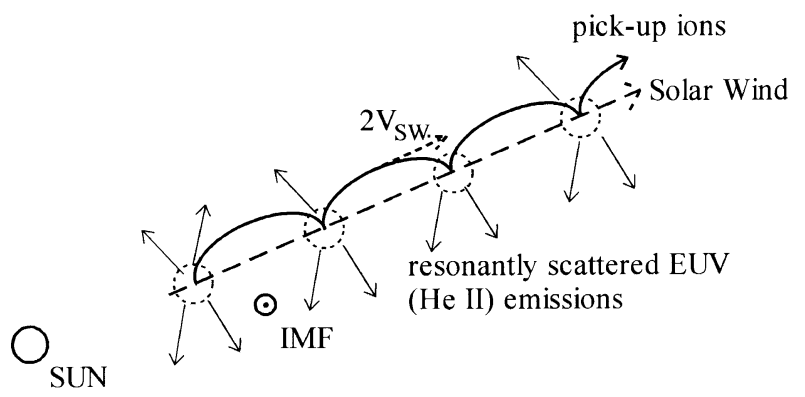

Fig. 1. Pick-up ions are flowing with the solar wind $\left(V_{\mathrm{SW}}\right.$ is the solar wind velocity). Inside the circles (dotted line) shown in the figure, the speed of the pick-up ions is slow and can resonantly scatter the solar EUV emissions. 


\section{Instrumentation}

\subsection{Sensor design}

The XUV is an X-ray ultraviolet scanner designed to detect He I $(58.4 \mathrm{~nm})$ and He II $(30.4 \mathrm{~nm})$ emissions. Plate 1 is a picture of the separated sensor elements, i.e., the hood, the telescope, and the pre-amplifier with the high-voltage (HV) supply unit. Plate 2 is a picture of the XUV installed on NOZOMI. It is mounted on the side panel looking downward. Figure 2 gives the cut view of the scanner. The telescope is thermally insulated from the spacecraft. It has an inclination of $30^{\circ}$ to the side panel. The telescope consists of a multi-layer coated mirror designed to focus the EUV emission and filters and microchannel plates (MCP) to amplify the signal.

The incident light coming into the telescope is reflected by the mirror and is focused on the MCP. A Mo/Si multilayer coated mirror (multi-layer period: $16.5 \mathrm{~nm}$; thickness of Mo: $4.1 \mathrm{~nm}$; pair number: 20; mirror surface: $5 \mathrm{~nm}$ Silicon) reflects preferentially $30.4 \mathrm{~nm}$ EUV light. It also reflects longer wavelength emissions above $50 \mathrm{~nm}$ as well. We need to detect only $30.4 \mathrm{~nm} \mathrm{He}$ II and $58.4 \mathrm{~nm} \mathrm{He}$ I emissions. Thus, the emissions above such wavelengths are

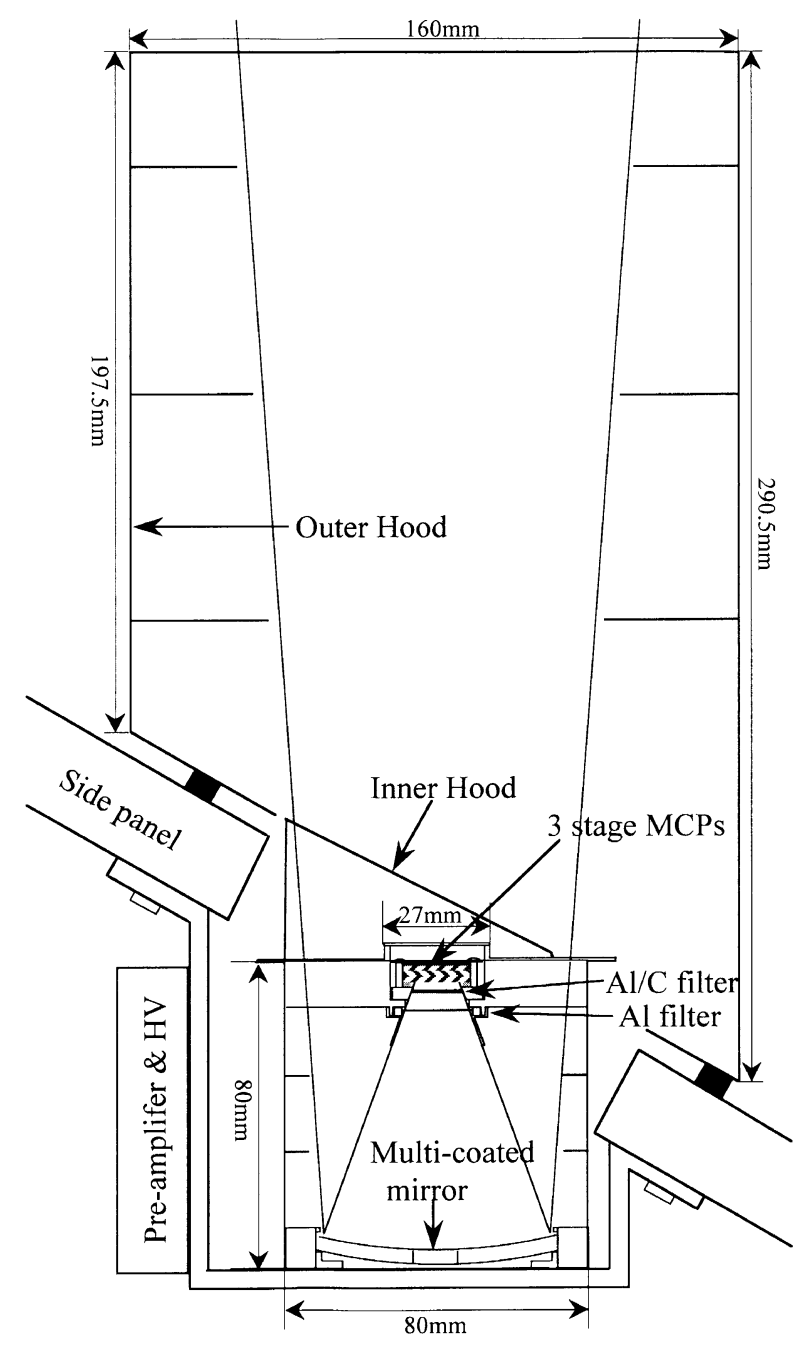

Fig. 2. A cross-section of the XUV scanner is illustrated. The scanner consists of a mirror, filters, MCP, and telescope tube and hoods. effectively attenuated by the filters.

Two filters are set in front of the MCP. They attenuate unnecessary EUV emissions up to visible light, e.g., O II emission at $83.4 \mathrm{~nm}$ and Lyman $\alpha$ emission at $121.6 \mathrm{~nm}$. The front Al filter protects the MCP from direct incident sunlight in a contingent case. This situation may happen only in a limited brief period at the reorientation of the spacecraft. The rear Al-C filter is separated into 2 areas (Plate 3). One half of it (Area A) has a $30.0 \mathrm{~nm}$-carbon coating on aluminum film and attenuates emissions with wavelengths longer than $40 \mathrm{~nm}$. The other part (Area B) is a bare aluminum film, not coated with carbon, which passes both $30.4 \mathrm{~nm}$ and $58.4 \mathrm{~nm}$ EUV emissions. The thickness of the filters is given in Table 1.

Three-stage MCP is used for signal detection. The first stage of the MCP is coated with CsI to increase the quantum efficiency of photon detection up to $15 \%$ at $30.4 \mathrm{~nm}$ (bare MCP has only $5 \%$ quantum efficiency at this wavelength). The gain is $10^{7}$ when $2.9 \mathrm{kV} \mathrm{HV}$ is applied between the MCP IN and MCP OUT electrodes.

To simplify the instrument system, we have employed a multi-anode system instead of a resistive anode for 2-D imaging. The signals are picked up by 4 anodes behind the MCP

Table 1. Filter thickness.

\begin{tabular}{cccc}
\hline Front & & Al & $129.9 \mathrm{~nm}$ \\
\hline \multirow{2}{*}{ Rear } & Area-A & Al & $142.9 \mathrm{~nm}$ \\
& & C & $30.8 \mathrm{~nm}$ \\
\cline { 2 - 4 } & Area-B & Al & $142.9 \mathrm{~nm}$ \\
& & C & - \\
\hline
\end{tabular}

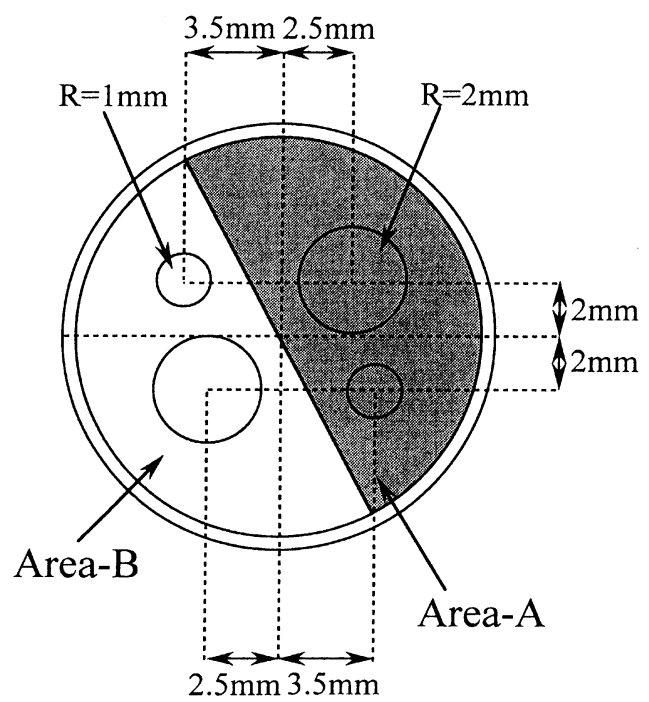

Fig. 3. The rear filter and anode configuration from the rear. (MCP is put between the filter and the anode, not illustrated here.) The filter is separated into 2 parts. Area A cuts the emissions over $40 \mathrm{~nm}$. Area B passes both $30.4 \mathrm{~nm}$ and $58.4 \mathrm{~nm}$ emissions. Small anodes have 1-mm radii and large anodes have $2-\mathrm{mm}$ radii, respectively. A pair of small and large anodes in Area A is used to detect the 30.4-nm EUV emission. Another pair in Area B detects the sum of the 30.4-nm and 58.4-nm EUV emissions. 


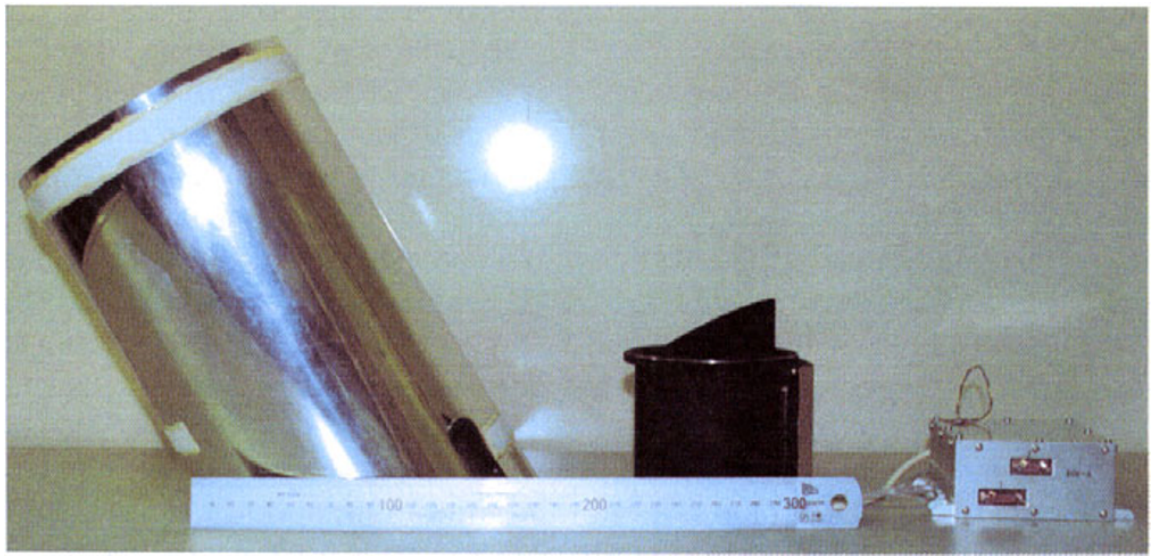

Plate 1. Separated XUV sensor parts. Outer hood (left side), telescope (middle), and the pre-amplifier circuit with the HV supply unit (right side).

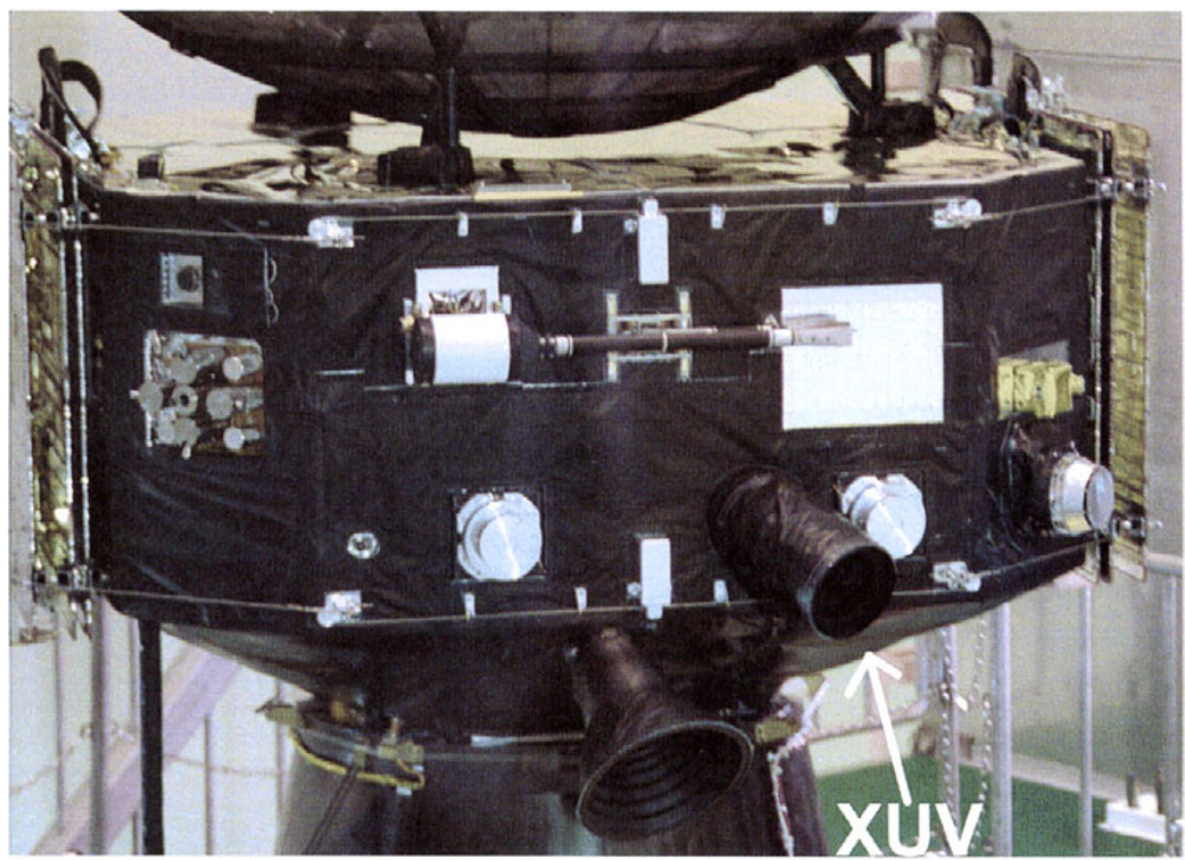

Plate 2. XUV sensor mounted on the side panel of NOZOMI.

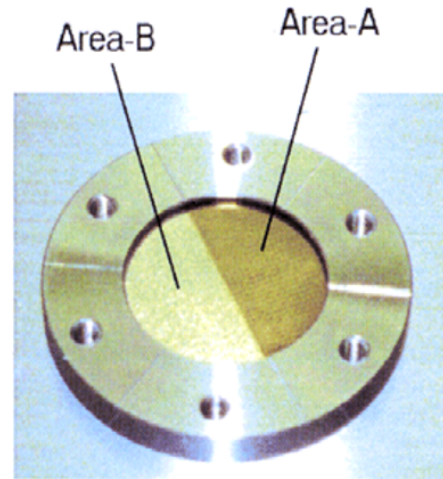

Plate 3. Rear filter. Half of the filter is coated with carbon.
(Fig. 3). Two of them have 1-mm radii (hereafter small anodes) and the other two have 2-mm radii (hereafter large anodes) which correspond to $6.1 \times 10^{-4}$ str. and $2.5 \times 10^{-3}$ str. field of view, respectively. A pair of small and large anodes in Area A are mainly used to detect the $30.4 \mathrm{~nm}$ EUV emission, and the other pair in Area B is used to detect the sum of the $30.4 \mathrm{~nm}$ and $58.4 \mathrm{~nm}$ EUV emissions. Subtracting the He II emission measured by the anodes in Area A from those in Area B gives the He I emission when the He I and II emissions are comparable.

The signals from the anodes are converted to the digital signals by the pre-amplifier (AMPTEK A111) and are counted in the control electronics called PAX (PSA and XUV control electronics). The data are edited by a CPU (HITACHI H32 micro-processor) and sent to the telemetry. Pre-amplifiers and the HV supply unit are put aside of the telescope to min- 


\section{Reflectivity of Multilayer-coated Mirror}

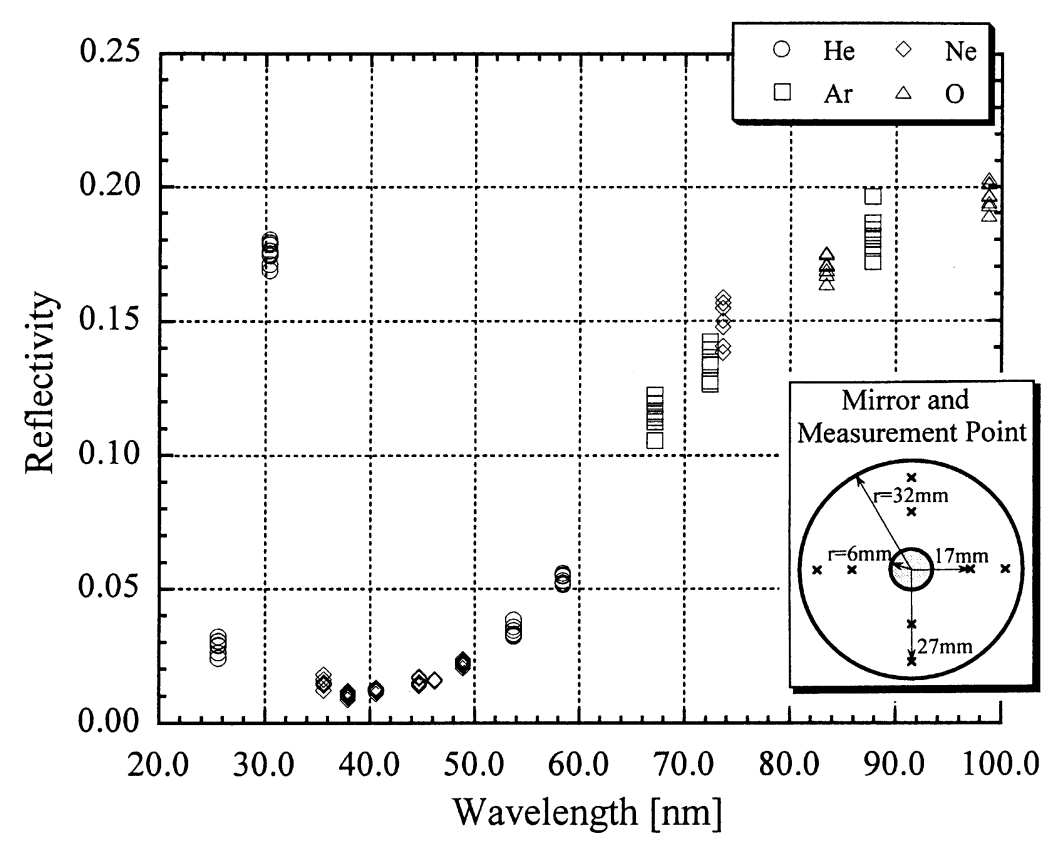

Fig. 4. Measured mirror reflectivity at the wavelength from 20 to $100 \mathrm{~nm}$. Measurements are given at 8 different points on the mirror as illustrated.

Table 2. Specification of the XUV scanner.

\begin{tabular}{lc}
\hline Size (Incl. pre-Amp. and HV unit) & $95 \mathrm{~mm} \phi \times 177 \mathrm{~mm}$ \\
Mirror aperture & $60 \mathrm{~mm} \phi$ \\
Focal length & $70 \mathrm{~mm}$ \\
MCP aperture & $10 \mathrm{~mm} \phi$ \\
View angle & \\
Small anode & $6.1 \times 10^{-4} \mathrm{str}$. \\
Large anode & $2.5 \times 10^{-3} \mathrm{str}$. \\
Weight & $870 \mathrm{~g}$ \\
Power consumption & $3.5 \mathrm{~W}$ \\
\hline
\end{tabular}

imize the signal cable length.

Adding to these basic telescope elements, we put a hood at the top of the telescope to attenuate stray light reflected by the star scanner (SSC) and the thermal plasma analyzer (TPA) sensor, which are mounted on the same side panel of the spacecraft. This hood consists of inner and outer parts. The inner part is directly attached to the telescope, while the outer part is attached to the spacecraft side panel.

As the sensor's field of view rotates around the spin axis, it makes a 1-D image in each spin. Summing up these 1-D images along the spacecraft orbit, we have 1 (possibly 2) 2$\mathrm{D}$ images in each orbit. We are aware of the poor temporal resolution of this image. The specifications of the XUV scanner are summarized in Table 2.

\subsection{Calibration}

The sensor was calibrated at the EUV calibration facility at the University of Tokyo. It consists of a vacuum cham- ber in which samples are set on a motion table, a discharge chamber, and a collimator. The most uncertain factor of the calibration is the absolute sensitivity of the MCP. We calibrated the sensitivity of another MCP (not the flight model) by comparing the photon count with a well-calibrated photo diode at the SOR facility in Okazaki, Japan. Then, we used this MCP as a standard for calibration at our EUV calibration facility. Details are published elsewhere (Yoshikawa et al., submitted to Res. Sci. Instrum.).

Figure 4 shows the reflectivity of the measured mirror. Measurements are given at 8 evenly distributed points all over the mirror surface (also shown in the figure). Particular line emissions of helium, argon, neon, and oxygen gas in the discharge chamber are used for the calibration. Thus, the data points are scattered in the wavelength. Maximum reflectivity $(17-18 \%)$ is achieved at $30.4 \mathrm{~nm}$. The reflectivity at $58.4 \mathrm{~nm}$ is $5-6 \%$.

The top panel of Fig. 5 shows the measured transmittance of the front $\mathrm{Al}$ filter, and the bottom panel shows that of the rear Al-C filter from 20 to $100 \mathrm{~nm}$. The transmittance in Area $\mathrm{A}$ is $18 \%$ at $30.4 \mathrm{~nm}$ and $0.7 \%$ at $58.4 \mathrm{~nm}$. The transmittance in Area B is $35 \%$ at $30.4 \mathrm{~nm}$ and $15 \%$ at $58.4 \mathrm{~nm}$.

After calibrating the quantum efficiency of the MCP, it was $15 \%$ at $30.4 \mathrm{~nm}$ and $20 \%$ at $58.4 \mathrm{~nm}$. The dark-noise count level of the MCP was $2 \mathrm{cps} / \mathrm{cm}^{2}$ when $3.0 \mathrm{kV} \mathrm{HV}$ was applied in the basement of the EUV calibration facility.

As the payload on NOZOMI is limited, we do not have an onboard calibration source. The EUV star may be used as a calibration source in the EUV star catalogue from the EUVE satellite. Another set of MCP is kept in our laboratory under the same conditions as onboard, i.e., in pure nitrogen gas before the spacecraft was launched and in the vacuum chamber after the launch. We are calibrating this set of MCP 

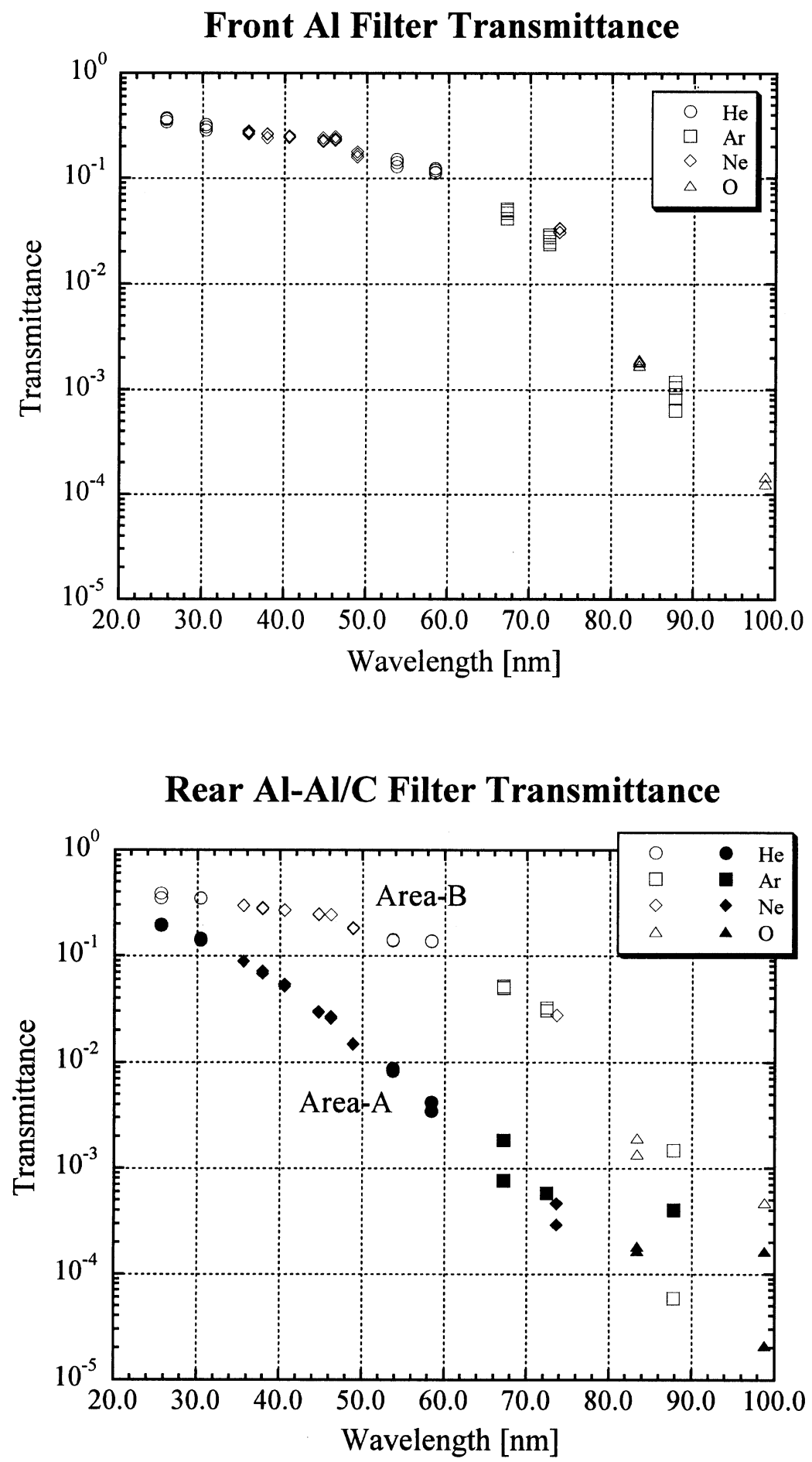

Fig. 5. (Top) Front Al filter transmittance at the wavelength from 20 to $100 \mathrm{~nm}$. (Bottom) Rear Al-C filter transmittance.

and evaluating the temporal change of the quantum efficiency of the MCP onboard.

\section{Expected Intensity of the Resonantly Scattered EUV Emissions}

In this section, we evaluate the He I and II emissions around Mars. We did not treat this as a radiation transfer problem but as a single scattering problem. Concerning helium ions around Mars and the earth, the optical thickness is thin in most cases and can be treated as a single scattering problem. As regards helium gas, the optical thickness may be thick, and it depends on the abundance of helium gas on Mars, which has not been determined yet. With the helium density profile which we have assumed and describe later, the optical thickness is less than 1 above $1000 \mathrm{~km}$ from the Martian surface. In this case, our evaluation is justified in most of the region.

The intensity of the scattered emission per unit solid angle, 
Table 3. $g$-factors of the He I and II emissions at Mars orbit.

\begin{tabular}{ccccccc}
\hline $\begin{array}{c}\text { Emission } \\
(\mathrm{nm})\end{array}$ & $f_{12}$ & $\begin{array}{c}\pi F \\
\left(\mathrm{~cm}^{-2} \mathrm{sec}^{-1} \mathrm{~nm}^{-1}\right)\end{array}$ & $\begin{array}{c}\text { Temp. } \\
(\mathrm{K})\end{array}$ & $\begin{array}{c}\Delta \lambda_{\mathrm{D}} \\
(\mathrm{nm})\end{array}$ & $\begin{array}{c}\sigma \\
\left(\mathrm{cm}^{2}\right)\end{array}$ & $\begin{array}{c}g \text {-factor } \\
\left(\mathrm{s}^{-1} \mathrm{particle}^{-1}\right)\end{array}$ \\
\hline He I & 0.2762 & $1.8 \times 10^{11}$ & 400 & $2.504 \times 10^{-4}$ & $1.880 \times 10^{-13}$ & $1.5 \times 10^{-5}$ \\
$(58.433)$ & & & 750 & $3.429 \times 10^{-4}$ & $1.373 \times 10^{-13}$ & $1.5 \times 10^{-5}$ \\
& & & 1000 & $3.959 \times 10^{-4}$ & $1.189 \times 10^{-13}$ & $1.5 \times 10^{-5}$ \\
\hline He II & 0.4162 & $2.5 \times 10^{11}$ & 400 & $1.302 \times 10^{-4}$ & $1.473 \times 10^{-13}$ & $8.5 \times 10^{-6}$ \\
$(30.378)$ & & & 750 & $1.782 \times 10^{-4}$ & $1.076 \times 10^{-13}$ & $8.5 \times 10^{-6}$ \\
& & & 1000 & $2.058 \times 10^{-4}$ & $9.316 \times 10^{-14}$ & $8.5 \times 10^{-6}$ \\
\hline
\end{tabular}

$f_{12}$ (oscillator strength) is from Meier (1991).

$I$, is given in the following equation:

$$
4 \pi I=10^{-6} p(\theta) g \int_{\mathrm{LOS}} n d s .
$$

The integral gives the column density, i.e., the density integrated along the line of sight (LOS). The coefficient $g=$ $\pi F \sqrt{\pi} \Delta \lambda_{\mathrm{D}} \sigma$ is the so-called $g$-factor, where $\pi F$ is the solar irradiance, $\Delta \lambda_{\mathrm{D}}$ is the Doppler width, and $\sigma$ is the line-center scattering cross section (Meier, 1991). The factor $p(\theta)$ is the scattering phase function. It is $p(\theta)=1+\frac{1}{4}\left(\frac{2}{3}-\sin ^{2} \theta\right)$ when the scattering is caused by hydrogen gas (Brandt and Chamberlain, 1959). Since this has not been determined yet with helium gas and ions, we assume that the scattering is isotropic, i.e., $p(\theta)=1$. The intensity of scattered emission, $4 \pi I$, is expressed with the unit Rayleigh, where 1 Rayleigh $=10^{6}$ photons $/ \mathrm{cm}^{2} / \mathrm{sec}$.

The $g$-factors of He I and He II emissions at Mars orbit are given in Table 3 . We assume a solar maximum condition as NOZOMI starts observation around Mars in October, 1999, which should be just before the next solar maximum year of 2001. It is known that the solar irradiance $\pi F$ has a good correlation with the $F_{10.7}$ value (Torr et al., 1979). When we assume $F_{10.7}=200 \times 10^{-22} \mathrm{Wm}^{-2} \mathrm{~Hz}^{-1}$ at 1 A.U. at the solar maximum, the He I emission is 3 times and the He II emission is 2 times larger than in the case with $F_{10.7}=70 \times 10^{-22} \mathrm{Wm}^{-2} \mathrm{~Hz}^{-1}$ at the solar minimum. Multiplying these factors by $\pi F$ in the Table IV in Meier (1991), which are given at the solar minimum condition, we have the solar irradiance at solar maximum at 1 A.U. At Mars' orbit (1.52 A.U.), the solar irradiance is only $43 \%$ of that at 1 A.U. Multiplying this number, we get $\pi F$ at Mars' orbit at solar maximum in Table 3. The line-center cross section and the Doppler width are calculated based on the oscillator strength $f_{12}$ given in Table IV in Meier (1991) with 400, 750, and $1000 \mathrm{~K}$ gas temperature. The Doppler width of the solar emission is $1.252 \times 10^{-2} \mathrm{~nm}$ for He I and $6.508 \times 10^{-3} \mathrm{~nm}$ for He II, assuming $100 \mathrm{eV}$ coronal temperature.

The expected photon count, $N_{\mathrm{p}}$, is given by the following equations:

$$
\begin{gathered}
N_{\mathrm{p}}=(4 \pi I) \cdot k, \\
k=\frac{10^{6} \eta S \delta}{4 \pi},
\end{gathered}
$$

Table 4. Sensor sensitivity.

\begin{tabular}{ccc}
\multicolumn{3}{c}{ Small anode } \\
\hline & $k_{304}$ & $k_{584}$ \\
\hline Area-A & 1.3 & $9.5 \times 10^{-3}$ \\
Area-B & 2.5 & $2.9 \times 10^{-1}$ \\
\hline
\end{tabular}

\begin{tabular}{ccc}
\multicolumn{3}{l}{ Large anode } \\
\hline & $k_{304}$ & $k_{584}$ \\
\hline Area-A & 5.0 & $3.9 \times 10^{-2}$ \\
Area-B & 9.5 & 1.2 \\
\hline
\end{tabular}

Suffix of $k$ represents $30.4 \mathrm{~nm}$ and $58.4 \mathrm{~nm}$, respectively.

where $\eta$ is the efficiency of the photon detection of the scanner, i.e., the product of mirror reflectivity, filter transmittance, and MCP quantum efficiency, $S$ is the detection area of the scanner, and $\delta$ is the view angle of the XUV scanner in solid angles. The sensor sensitivity $k$ depends only on the sensor design, and $4 \pi I$ depends only on the EUV emission source. Table 4 gives the sensitivities of the small and large anodes in Area A and Area B for He I emission $\left(k_{584}\right)$ and He II emission $\left(k_{304}\right)$.

We do not expect a larger noise count due to the highenergy particles from the radiation belt or the cosmic ray because the MCP is well shielded by SUS304 and the aluminum case. Moreover, NOZOMI flies away from the radiation belt of the planets. The dark count rate $\left(2 \mathrm{cps} / \mathrm{cm}^{2}\right)$ corresponds to $0.06 \mathrm{cps}$ for small anodes and $0.25 \mathrm{cps}$ for large anodes. The He II emission of $5 \times 10^{-2}\left(3 \times 10^{-2}\right)$ Rayleigh competes with these dark counts in Area A (B). In other words, the signal to noise ratio is better than 1 when we treat emissions brighter than the above criterion. A similar criterion for the He I emission is 0.25 Rayleigh, with the anodes in Area B (anodes in Area A are not sensitive to the He I emission).

\subsection{EUV intensity from the Martian atmosphere and ionosphere}

The distribution of helium in Mars has not been established in previous observations. Brightness needs to be evaluated 
assuming the density profile.

Helium gas distribution in the Martian atmosphere is assumed to have a barometric density profile with a $420 \mathrm{~km}$ scale height (corresponding to $750 \mathrm{~K}$ gas temperature) and a density of $10^{6} \mathrm{~cm}^{-3}$ at the ground level.

It is not easy to estimate the helium ion distribution on Mars. Barabash and Norberg (1994) reported the pick-up ion density at the Phobos orbit, but this number should be much less than that in the ionosphere and can not be employed here. Comparing the model of the helium gas density profile in Mars with the density profile of helium gas on earth, we find that the former one is 0.1 times as much as that on earth at an altitude of $1000 \mathrm{~km}$. Therefore, we assume the same ratio on helium ions, i.e., the helium ion density in Mars is assumed to be 0.1 of that on earth, which is $10^{3}-10^{4} \mathrm{~cm}^{-3}$ depending on the solar activity. We took the largest number $10^{3} \mathrm{~cm}^{-3}$ as the Martian helium ion density in the ionosphere from an altitude of $500 \mathrm{~km}$ to $1700 \mathrm{~km}$. The upper limit of the ionosphere is taken to be the magnetopause distance from the surface of Mars at the terminator. The density profiles of helium gas and ions are shown in Fig. 6. The He I and He II emissions from Mars are evaluated with Eq. (1). Figures 7(a) and (b) show the calculated He I and He II emissions as a function of the line of sight (LOS) distance from Mars' center, respectively. LOS is assumed to be parallel to the sun-Mars line. The He I emission gives its maximum $\left(4.1 \times 10^{3}\right.$ Rayleigh $)$ at the Martian rim. The emission exceeds the noise count level $\left(5 \times 10^{-2}\right.$ Rayleigh) even at $2 \mathrm{R}_{\mathrm{m}}$ (Mars' radii) from the center. On the other hand, the He II emission is faint. It is almost constant (2.9 Rayleigh) between 1 and $1.45 \mathrm{R}_{\mathrm{m}}$ and falls below the noise count level beyond $1.5 \mathrm{R}_{\mathrm{m}}$.

Figures 8(a) and (b) show the photon count rates corresponding to the He I and II emissions to the large anodes in Area A and Area B, respectively. The noise count level of MCP $(0.25 \mathrm{cps})$ is also shown. From these figures, we learn that the He I emission is dominant around Mars and

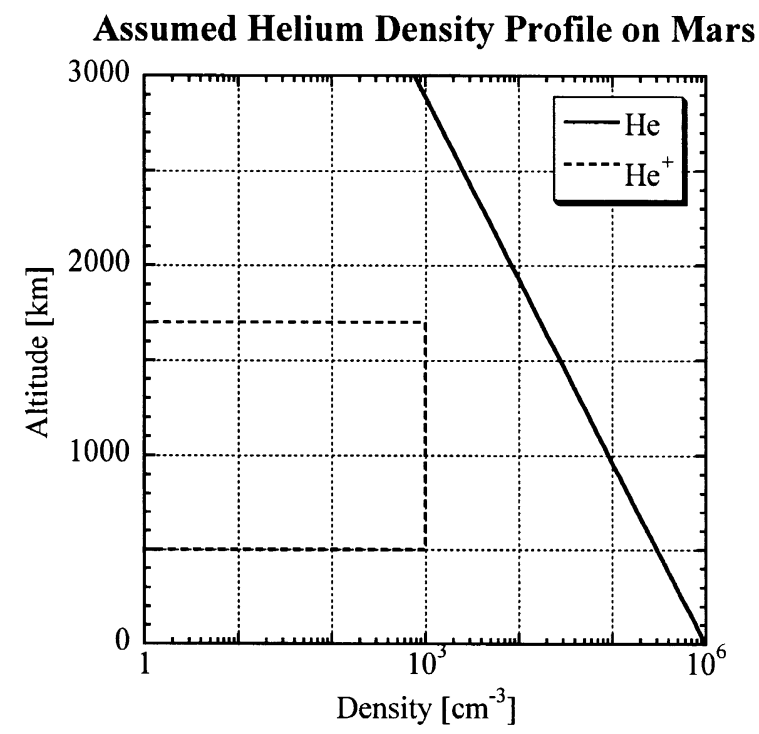

Fig. 6. Density profile of helium gas and ions in Mars assumed for the estimation.
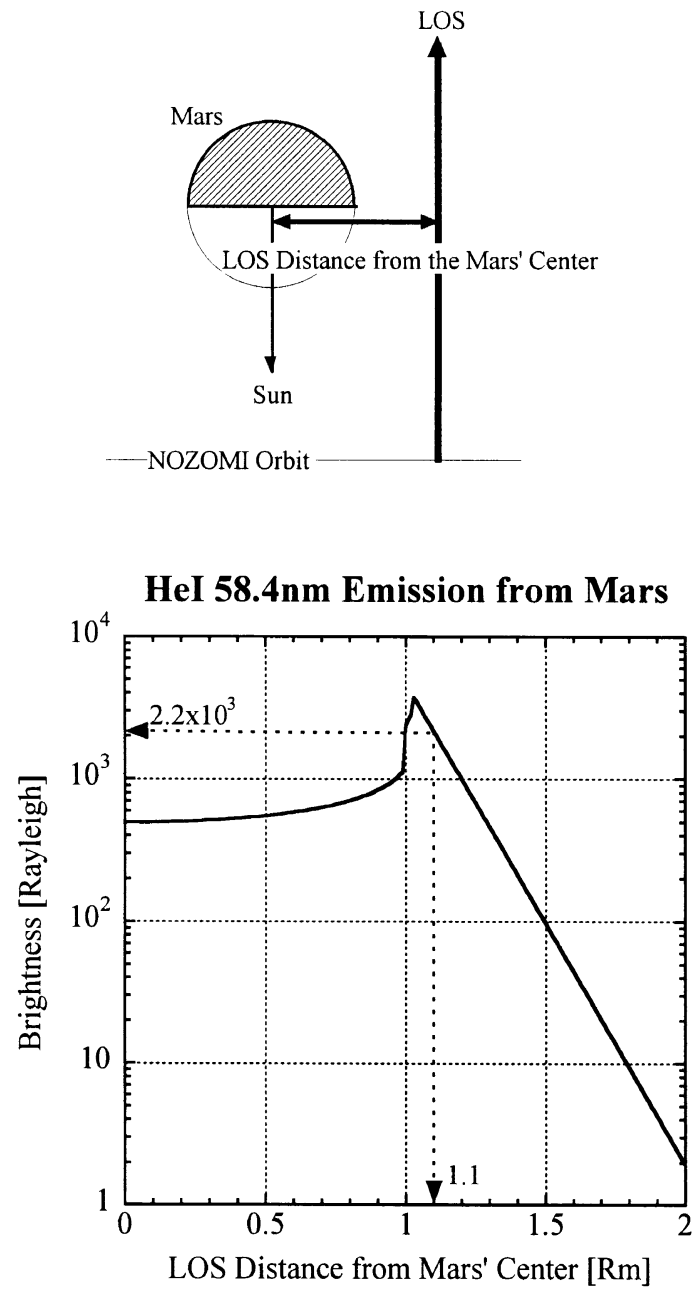

(a)

HeII 30.4nm Emission from Mars

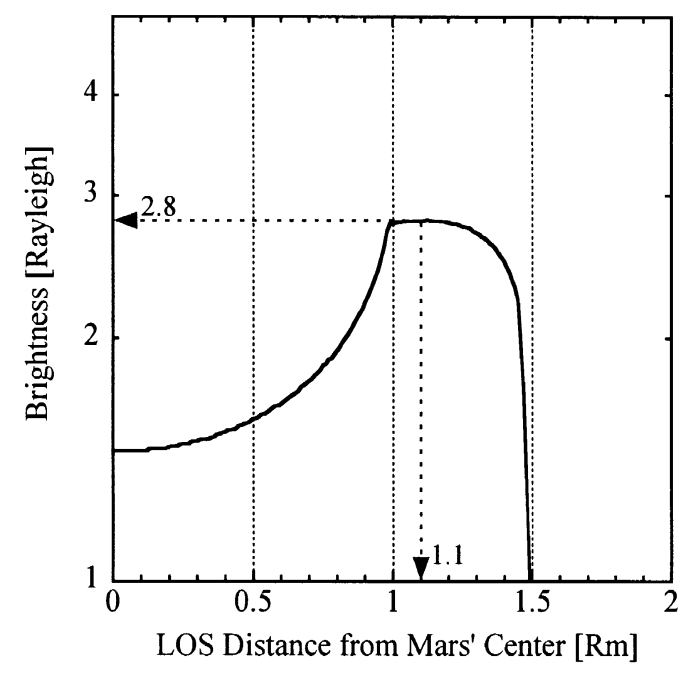

(b)

Fig. 7. Estimated He I emission (a) and He II emission (b) from Mars' atmosphere as a function of a LOS distance from Mars' center. 


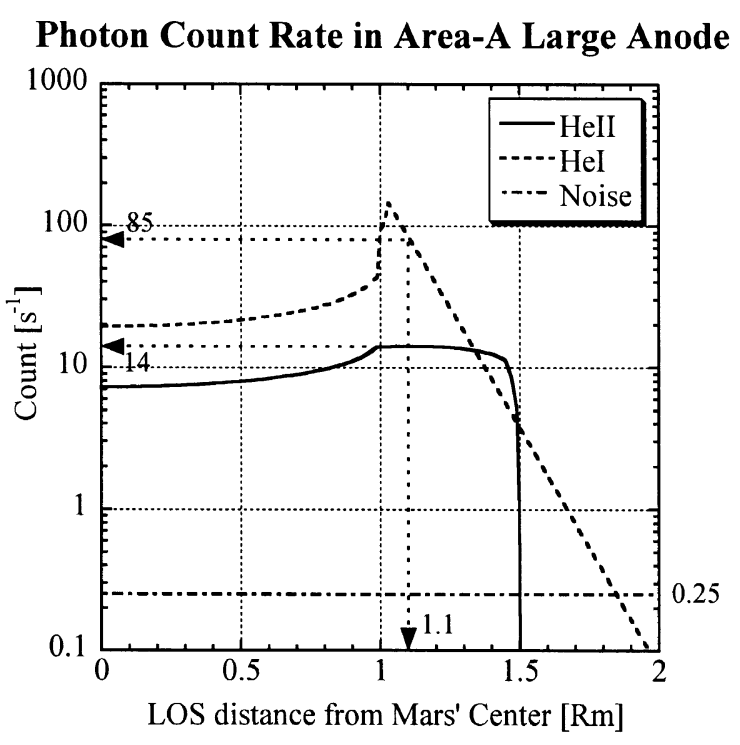

(a)

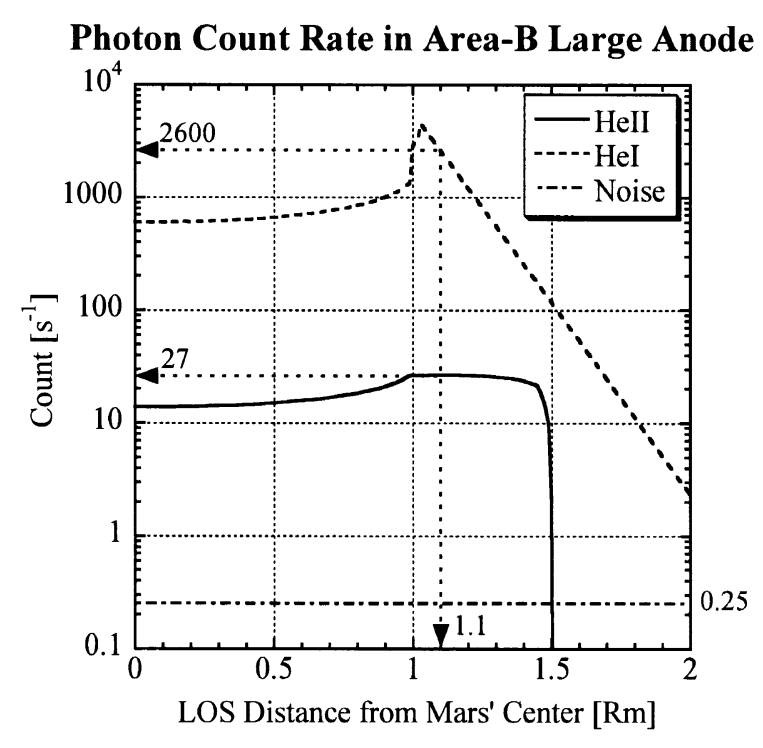

(b)

Fig. 8. Estimated photon count rate corresponding to $\mathrm{He}$ I and $\mathrm{He}$ II emissions in Area A large anode (a) and Area B large anode (b) as a function of a LOS distance from the Mars' center.

the separation of the He I and II emissions is not so easy. In Area B, most of the count is attributed to the He I emission. The helium gas density profile was obtained from this data. Next, we need to separate the He II emission from the He I emission from the data in Area A. Statistics require that the accumulation time $T$ satisfy the following equation to separate the He II from the He I emission:

$$
\sqrt{\left(H e I_{\mathrm{A}}+H e I I_{\mathrm{A}}\right) T} \ll H e I_{\mathrm{A}} \cdot T,
$$

where $\mathrm{HeI}_{\mathrm{A}}\left(\mathrm{HeII}_{\mathrm{A}}\right)$ is the $\mathrm{He} \mathrm{I}(\mathrm{He} \mathrm{II})$ emission count rate in Area A. The left-hand side term gives the relative statistical error of the accumulated count in Area A, while the right- hand side term gives the accumulated count attributed to the He II emission. For example, when the LOS distance is $1.1 \mathrm{R}_{\mathrm{m}}$ from Mars' center, $\mathrm{HeI}_{\mathrm{A}}$ is $85 \mathrm{cps}$, while $\mathrm{HeII}_{\mathrm{A}}$ is 14 cps. Using Eq. (4), we see that the accumulation time $T$ should be longer than $0.5 \mathrm{sec}$. If we sum up the data over $6^{\circ}$, $1 / 60$ data for each spin are useful. Then, we can separate the He II emission from the data in Area A when we accumulate the data for much longer than $30 \mathrm{sec}(0.5 \times 60 \mathrm{sec})$. Ten min. of accumulation time should be long enough to satisfy this condition.

\subsection{EUV intensity from the plasmasphere of the earth}

We have the opportunity to observe the earth's plasmasphere and magnetotail from the parking orbit around the earth. The He II emission is expected to be 0.1-20 Rayleigh from the plasmasphere (Williams et al., 1992). As the He I emission should come only from the vicinity of the earth, we may use the data from the anodes in Area B for He II observation. The expected count rate corresponding to 1 Rayleigh He II emission is $9.5 \mathrm{cps}$ with the large anode by using the $k_{304}$ in Table 4. The angular resolution corresponding to the large anode is $3.3^{\circ}$, which equals $0.2 \mathrm{Re}$ (earth radii) spatial resolution (scanning line width) when the spacecraft is located $7 \mathrm{Re}$ apart from the earth. Considering that the spin period is about $6.45 \mathrm{sec}$ in the parking orbit, we have $59 \mathrm{msec}$ in one spacecraft rotation if we need the same spatial resolution in the scanning direction, i.e., the pixel size is $0.2 \times 0.2 \mathrm{Re}$. The expected count rate corresponding to 1 Rayleigh is 0.6 for each pixel for 1 spin period. We thus conclude that data accumulation over several spin periods will yield a satisfactory image.

\subsection{EUV intensity from the magnetotail of the earth}

Let us assume the cold $\mathrm{He}^{+}$density in the tail region to be $0.1 \mathrm{~cm}^{-3}$ and the column length to be $40 \mathrm{Re}$. Since the $g$-factor of the He II emission at 1 A.U. is $9.2 \times 10^{-6}$ $\mathrm{s}^{-1}$ particle ${ }^{-1}$ at the solar minimum condition in 1998 (Table IV in Meier, 1991), the expected luminosity is $2.3 \times$ $10^{-2}$ Rayleigh. Unfortunately, the corresponding count rate is less than the dark-noise count rate. When NOZOMI is at the midnight sector and the observation is along the magnetotail, the column length is much longer, over $200 \mathrm{Re}$. The He II emission is 5 times brighter than in the previous case, and the expected signal count exceeds the dark-noise count level. We conclude that we will have many opportunities to detect He II emissions from the earth's magnetotail when the line of sight of the observation is along the magnetotail.

\section{Conclusion}

The XUV scanner designed to detect EUV emissions from helium gas and ions around Mars is on board Japan's Mars orbiter NOZOMI. It will provide data concerning the abundance and distribution of helium around Mars. In the parking orbit around the earth and in the transfer orbit to Mars, the XUV scanner will be operated for the study of the terrestrial plasmasphere and interplanetary EUV emissions.

Simultaneous observations with ion mass imager (IMI), neutral mass spectrometer (NMS), and ion spectrum analyzer (ISA) are important, because the XUV observation will give the global image of the plasma and neutral gas distribution, while the in situ measurements will give the qualitative number of these distributions, thus being complementary. 
Acknowledgments. This paper is dedicated to the memory of Tatsundo Yamamoto. We are grateful to H. Hayakawa, A. Nishida, and $\mathrm{K}$. Tsuruda for their help and encouragement in our experiment. We greatly appreciate the referees and the guest editor for their kind advise. We thank Meisei Co. Ltd. (electronics), Mitaka Koki Co. Ltd. (telescope), Nikon (multi-layer mirror), Hamamatsu Photonics (MCP), and Luxel Co. Ltd. (filter), for manufacturing each part of the XUV sensor.

\section{References}

Banks, P. M., A. F. Nagy, and W. I. Axford, Dynamical behavior of thermal protons in the mid-latitude ionosphere and magnetosphere, Planet. Space Sci., 19, 1053-1067, 1971.

Barabash, S. and O. Norberg, Indirect detection of the Martian helium corona, Geophys. Res. Lett., 21, 1547-1550, 1994.

Barabash, S., E. Kallio, R. Lundin, and H. Koskinen, Measurements of nonthermal helium escape from Mars, J. Geophys. Res., 100, $21307-$ 21316, 1995.

Brandt, J. C. and J. W. Chamberlain, Interplanetary gas, 1, hydrogen radiation in the night sky, Astrophys. J., 130, 670-682, 1959.

Krasnopolsky, V. A., S. Bowyer, S. Chakrabarti, G. R. Gladstone, and J. S. McDonald, First measurement of helium on Mars: Implications for the problem of radiogenic gases on the terrestrial planets, Icarus, 109, 337-351, 1994.
Luhmann, J. G. and L. H. Brace, Near-Mars space, Rev. Geophys., 29, 121$140,1991$.

Luhmann, J. G. and J. U. Kozyra, Dayside pickup oxygen-ion precipitation at Venus and Mars: Spatial distributions, energy deposition, and consequences, J. Geophys. Res., 96, 5457-5467, 1991.

Meier, R. R., Ultraviolet spectroscopy and remote sensing of the upper atmosphere, Space Sci. Rev., 58, 1-185, 1991.

Norberg, O., M. Yamauchi, R. Lundin, S. Olsen, H. Borg, S. Barabash, M. Hirahara, T. Mukai, and H. Hayakawa, The Ion Mass Imager on the Planet-B spacecraft, Earth Planets Space, 50, 199-206, 1998.

Sasaki, S. and E. Tajika, Degassing history and evolution of volcanic activities of terrestrial planets based on radiogenic noble gas degassing model, in Volatiles in the Earth and Solar System, AIP Conference Proceedings 341, edited by K. A. Farley, pp. 186-199, American Institute of Physics, 1995

Torr, M. R., D. G. Torr, R. A. Ong, and H. E. Hinteregger, Ionization frequencies for major thermospheric constituents as a function of solar cycle 21, Geophys. Res. Lett., 6, 771-774, 1979.

Williams, D. J., E. C. Roelof, and D. G. Mitchell, Global magnetospheric imaging, Rev. Geophys., 30, 183-208, 1992.

M. Nakamura (e-mail: mnakamur@sunep1.geoph.s.u-tokyo.ac.jp), K Yamashita, I. Yoshikawa, K. Shiomi, A. Yamazaki, S. Sasaki, Y. Takizawa, M. Hirahara, W. Miyake, Y. Saito, and S. Chakrabarti 